\title{
Accountability en Chile. Un Estudio Sociocrítico sobre Innovación Educativa y Control de la Docencia
}

\section{Accountability in Chile: A Critical Research about Educational Innovation and Control of Teachers' Work}

\author{
Javier Pascual Medina * \\ David Rodríguez Gómez
}

Universitat Autònoma de Barcelona, España

\begin{abstract}
El objetivo de este artículo es conocer, desde una perspectiva teórica basada en el conflicto y el poder, cómo los procesos de accountability en Chile tensionan a los docentes para adherirse al currículum central, y de qué manera afectan los procesos de innovación educativa. Hemos conducido un estudio desde un paradigma sociocrítico, con herramientas cualitativas y participativas, construyendo producciones narrativas con nueve docentes en la zona central de Chile. Los resultados muestran que los docentes se sienten atados a un sistema educativo de estándares muy rígidos, con un currículum central demasiado exhaustivo y un sistema de accountability que limita sus posibilidades de acción y, por lo tanto, sus posibilidades de realizar innovaciones educativas profundas. También muestran que este sistema ha permeado en un contexto discursivo, delimitando tanto la interpretación de los indicadores como las expectativas que se tienen sobre los docentes y, por lo tanto, su propia motivación a hacer cosas diferentes a las ya establecidas. Concluimos que la homogeneización de la tarea docente bajo propuestas neoliberales de organización de los centros educativos y desarrollo curricular supone no sólo una reducción notable de la autonomía docente, sino que limita la posibilidad de desarrollar procesos creativos en los centros educativos y, consecuentemente, dificulta la aparición de innovaciones que podrían llevar a mejoras educativas significativas. A su vez, se manifiesta la necesidad de que los docentes se empoderen, especialmente del currículum educativo, para retomar el control de su trabajo pedagógico.
\end{abstract}

Palabras Clave: Innovación educativa; Accountability; Control curricular; Poder; Conflicto.

\begin{abstract}
This article starts from a theoretical perspective based on conflict and power. The main goal is to know how Chilean educational accountability processes push teachers to stick to the national curriculum, and how they affect educational innovation processes inside their schools. We conducted a study from a sociocritic paradigm, with qualitative and participative tools. We constructed narrative productions with nine teachers from Chile. Results show that teachers feel tied to an educational system with rigid standards, an extremely exhaustive national curriculum and a stressful accountability system. These elements limit teacher's actions and possibilities to do deep educational innovations. At the same time, teachers from non-evaluated disciplines in this accountability system feel abandoned by their institutions, so they cannot take advantage of their freedom because of the lack of support to make their innovations sustainable. As well, results show that this system has permeated into a discursive context, delimiting the indicators interpretations, the social expectations over teachers and, therefore, their motivation to do things other than those stablished. We conclude that homogenization of teachers' tasks under neoliberal models reduce teachers' autonomy and limit creative process. This hinders the creation and adaptation of educational innovations that could bring the school to meaningful improvements. However, teachers need to empower themselves and their relationship with curriculum and take control of their own pedagogical work.
\end{abstract}

Keywords: Educational innovation; Accountability; Curriculum control; Power; Conflict.

*Contacto: jpascualmedina@gmail.com

issn: $1989-0397$

www.rinace.net/riee/

https://revistas.uam.es/riee
Recibido: 27 de julio de 2018

$1^{\text {a }}$ Evaluación: 26 de septiembre de 2018

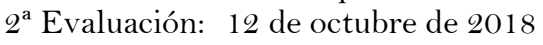

Aceptado: 15 de octubre de 2018 


\section{Introducción}

En los establecimientos educativos se presentan cambios constantemente, como pueden ser las aplicaciones de nuevas metodologías de enseñanza, la incorporación de materiales pedagógicos o nuevas tecnologías, la reestructuración de infraestructuras, las modificaciones en los organigramas o en las formas de coordinación directivas, los cambios de personal entre muchas otras (Margalef y Arenas, 2006). La innovación educativa se vincula a cualquier modificación intencional de actitudes, comportamientos, procedimientos o prácticas educativas (Rivas, 2000), entendiéndose, por lo tanto, como un proceso complejo que conlleva a alteraciones a partir de una situación inicial, siempre y cuando dicha alteración esté orientada desde su intención a un mejoramiento de dicha situación inicial (Murillo, 2002). Diferenciamos, antes de seguir adelante, de los conceptos de Reforma o Mejora, pues estos se refieren a cambios en el sistema educativo en su conjunto o cambios en las instituciones educativas respectivamente. Definimos una innovación educativa como un cambio directo en los procesos de enseñanza-aprendizaje, que está orientado al mejoramiento de la calidad de la educación desde su concepción (Murillo, 2002).

Uno de los puntos centrales para pensar en la innovación debe ser entender que su efecto sobre algo no sólo se da en una relación de simple causalidad, sino dentro de una red de interacción compleja donde todo lo modificado provoca a su vez otros ciclos de transformación (Cruz y Croda, 2017). Por lo mismo, la innovación se ha posicionado durante las últimas décadas como pieza clave de los procesos educativos, pues se ha comprendido que la educación necesita cambiar bajo una mirada holística y compleja (Sánchez Chacón, 2016). A la vez, sin embargo, los sistemas educativos buscan encaminar los cambios educativos de acuerdo a objetivos planteados para el desarrollo de un país, intentando balancear la diversidad y complejidad de sus múltiples contextos locales (Burns y Köster, 2016). Para esto, enmarcan y supervisan la educación mediante una serie de tecnologías, donde dos ocupan una posición predominante: la definición de un currículum central y el establecimiento de procesos de accountability.

Se puede considerar que el currículum es la piedra angular que guía los procesos de enseñanza y aprendizaje, pues es lo que define qué, cómo, quién, a quién y para qué se enseñan determinados contenidos y se forman determinadas habilidades. El currículum define los estándares de aprendizaje, que es lo que define lo que se espera que todo estudiante dentro de un país o una región debe saber o ser capaz de hacer (Tejada, 2005). La definición de estándares de aprendizaje debiera, por lo tanto, contribuir en clarificar y hacer explícitas las expectativas de aprendizaje, alinear dichas expectativas con las demandas sociales, guiar la enseñanza, fomentar compromisos de equidad, proveer criterios comunes sobre el avance de los estudiantes, facilitar la comunicación entre los diversos actores educativos, enfatizar los objetivos finales de las escuelas y las políticas públicas, e identificar estudiantes con necesidades de apoyo (OCDE, 2013).

Para monitorear el cumplimiento efectivo de este currículum en los establecimientos educativos, se han instalado en los sistemas educativos diversos modelos de accountability basado en los resultados de aprendizaje de los estudiantes. Los defensores de estas políticas a nivel internacional, desde una mirada neoliberal del sistema educativo, señalan que estos modelos responsabilizan a las escuelas por los resultados de aprendizaje de los estudiantes, empoderan a estudiantes y familias para exigir buenos resultados, y se aprovecha como un método de retroalimentación para que los docentes puedan mejorar 
sus prácticas maximizando el potencial educativo del sistema (Puryear, 2006). Sin embargo, varias observaciones se han hecho desde los enfoques más críticos, entre ellas que mantienen una política de arriba hacia abajo disfrazada de una política de abajo hacia arriba, desprofesionalizan y tecnifican la profesión docente, y si bien no ha sido posible demostrar un efecto positivo de este tipo de reformas en los aprendizajes de los estudiantes, si se han encontrado efectos negativos en aspectos como la inclusión, la segregación y la calidad de vida de los docentes y directivos (Casassus, 2010). Au (2009) lo define como un aparato tecnológico que sólo intenta mantener el modelo neoliberal, abogando por la categorización y ordenamiento eficiente de las personas, objetivizando y mercantilizando a las personas, e incrementando la capacidad de las autoridades de controlar y supervisar los procesos educativos. Ante esto, se lograría una relación de poder oculta en la que se logra tener un control tanto técnico como discursivo de la acción docente. Desde el punto de vista técnico, principalmente existe una relación de poder en torno al currículum, manteniendo un control del contenido, forma y método -es, decir, qué cosas, bajo qué enfoques y con qué metodologías se enseña. Adicionalmente, desde el punto de vista discursivo, mantienen un control sobre la identidad de los docentes y de los estudiantes, pues definen claramente cuál es el rol de cada uno, y cuáles son las formas adecuadas o inadecuadas de acción dentro de los procesos de enseñanza y aprendizaje (Au, 2009).

Estos dispositivos de control, por lo tanto, son capaces de mantener cierta homogeneidad de las prácticas educativas, reduciendo el currículum de los establecimientos y, por ende, las posibilidades de cambio educativo. Giles y Hargreaves (2006) plantean que los contextos rígidos debilitan las culturas de innovación de los establecimientos educativos, porque llegan a estandarizar los procesos de cambio de tal manera que dejan poco espacio para que los docentes aprendan durante estos procesos y los desmotivan.

El objetivo de este estudio es conocer cómo los procesos de accountability en Chile tensionan a los docentes para adherirse el currículum central, y de qué manera afectan los procesos de innovación educativa dentro de los establecimientos educativos del país.

En Chile se proporciona desde el nivel central instrumentos curriculares y recursos educativos que contribuyen tanto al trabajo en aula, como a la evaluación de los aprendizajes de los estudiantes, bajo un set de herramientas que forman parte del Sistema Curricular (MINEDUC, 2017). Se trata de un currículum central exhaustivo que abarca todas las disciplinas, define qué deben aprender los estudiantes y propone cómo se puede aplicar, apoyar y monitorear dentro de las escuelas, aunque a su vez deja una cantidad delimitada de horas de libre disposición para que las escuelas puedan complementarlo como prefieran.

A la par de la centralización del currículum, desde principios de los años 90 en Chile se han intensificado políticas de responsabilización por desempeño de las escuelas, o como son normalmente llamadas, políticas de accountability. Éstas políticas introducen los principios del mercado y la competencia en educación, estableciendo mediciones basadas en los estándares establecidos por el currículum central, que permitirían informar a las familias sobre la calidad de las escuelas y darles herramientas para optimizar su elección (Falabella y de La Vega, 2016). Accountability significa, básicamente, fijar la responsabilidad por las propias acciones y los resultados de dichas acciones (McMeekin, 2006), y se ha instalado a través de reformas basadas en estándares en muchos países 
occidentales, sin estar exento de un debate que transita entre lo político y lo académico (Espinola y Claro, 2010).

En Chile existen al día de hoy dos evaluaciones estandarizadas a nivel nacional que componen un fuerte sistema de accountability. El más importante es el Sistema de Medición de la Calidad de la Educación (SIMCE), el cual fue creado en 1988 y tomado por el Ministerio de Educación en 1992. El año 2012 la responsabilidad de analizar los resultados fue traspasada a la Agencia de Calidad de la Educación, con el fin de desmarcarlos de los tintes ideológicos de cada gobierno. Se trata de una prueba censal - es decir, se aplica en todos los establecimientos del país - y hasta el año 2012 se evaluaba todos los años a $4^{\mathrm{a}}$ básico, y se alternaba cada año $8^{\circ}$ básico u $2^{\circ}$ medio. En 2013 se añadieron pruebas censales para $2^{\circ}$ y $6^{\circ}$ básico, y se comenzó a aplicar anualmente en los otros niveles, idea que fue ampliamente cuestionada, por lo que el año 2016 se anunció una reducción de estas evaluaciones. Hoy se aplica anualmente en $4^{\circ}$ básico y $2^{\circ}$ medio, se alternan anualmente $6^{\circ}$ y $8^{\circ}$ básico y la de $2^{\circ}$ básico es de carácter muestral. Las censales abarcan las disciplinas de Lenguaje, Matemáticas, Ciencias Naturales y Ciencias Sociales, y para el año 2020 se espera tener además una prueba específica de competencias generales de alumnos de enseñanza técnico profesional.

Por otro lado, existe la Prueba de Selección Universitaria (PSU), concebida por el Consejo de Rectores de Universidades de Chile (CRUCH) el año 2003, como sucesora de la Prueba de Aptitud Académica (PAA). La PSU se aplica a todos los egresados de la enseñanza secundaria que quieran acceder a la educación superior, a quienes ordena de acuerdo a los puntajes para seleccionar quiénes llenarán las plazas disponibles en las diversas carreras. La PSU no tiene un objetivo directo de accountability, pero se ha utilizado con este fin por gobiernos y medios de comunicación para justificar políticas y prácticas en educación.

La política curricular chilena resulta altamente prescriptiva, por lo que la formación opera como un estándar que define con un alto nivel de especificación los saberes mínimos necesarios y alcanzables para todos los estudiantes, lo que limita de forma importante la labor docente (Assaél, Albornoz y Caro, 2018). A su vez, las políticas de accountability regulan dicha prescripción curricular, manteniendo un control rígido sobre los procesos educativos oculto bajo una imagen de autonomía y libertad de parte de las escuelas (Casassus, 2010). Con todo esto, es posible intuir que los procesos de cambio e innovación educativa se verían restringidos o limitados por un sistema educativo que aboga por la regulación y el control de la enseñanza.

\section{Enfoque Teórico}

Comprendidos los conceptos principales y el contexto en el que se enmarca el estudio, el siguiente apartado resume brevemente el enfoque o paradigma teórico desde el cual nos posicionamos para este estudio. Es importante destacar que el paradigma escogido y la metodología coherentemente seleccionada para este artículo no responden a los estándares tradicionales de investigación y divulgación, pues exigen que esta sección se centre en los marcos generales que orientan el estudio, dejando una revisión más detallada del estado de la cuestión y de las categorías de análisis en la sección de resultados. Todo esto situar el conocimiento experiencial y los discursos de los sujetos participantes a la par de los conocimientos académicos, y no al alero de éstos, como suele hacerse. Más adelante se retomará este punto para explicarlo y justificarlo con más antecedentes. 
Para empezar, nos posicionamos desde un paradigma sociocrítico. Elegimos este enfoque dada la necesidad de ser coherentes con las intenciones propias de la innovación educativa, es decir, con la transformación y el cambio educativo. También es un estudio que requiere comprender a los docentes como los principales agentes de cambio y, por lo tanto, piezas clave en las relaciones de poder que inciden en los procesos de innovación educativa. La enseñanza, bajo este enfoque, se ve como una actividad crítica para la emancipación personal y colectiva, y no tiene por finalidad solo dar a conocer el mundo, sino transformarlo. El diálogo es la base de la acción educativa, pues ilumina problemas y busca soluciones colectivas y democráticas (Tejada, 2005), pero al mismo tiempo se configura como la manifestación de un conflicto entre agentes que tienen distintos intereses y necesidades. La innovación, desde esta perspectiva, requiere de docentes críticos, empeñados en construir innovaciones teóricas, buscando verdades dialogadas en comunidades discursivas y prácticas reflexivas (Escudero, 2014).

Siguiendo esta línea, entendemos la innovación educativa desde una perspectiva dialéctica. A través de los años, el cambio ha sido estudiado desde diversas corrientes de pensamiento. La perspectiva dialéctica nace en respuesta a modelos tradicionales de cambio que estaban basados en perspectivas estructural-funcionalistas (Kezar, 2001), que mostraban una visión bastante determinista y conservadora que concebían a la sociedad como un organismo armónico, compuesto de acciones que están determinadas por sistemas de valores y conciencias colectivas que preceden a los actores y que tienen una finalidad común orientada a la estabilidad social, y donde el cambio siempre se presenta de forma lineal, estable y determinado por el contexto (Durkheim, 1987; Parsons, 1999).

Derivada de las Teorías del Conflicto, la perspectiva dialéctica sostiene que diferentes actores se conforman como agentes, individuales o colectivos, que defienden sus intereses, muchas veces en detrimento de los intereses de otros agentes, lo que lleva al enfrentamiento de ambas partes. El conflicto puede entenderse simplemente como la consecuencia de una situación de divergencia que sostienen personas o grupos sociales al poseer intereses o valores diferentes (Silva, 2008), y no es sólo la norma en los procesos sociales, sino la energía impulsora del cambio y evolución social, y debe concebirse como un elemento existencial que atañe a la vida de cada individuo, no solamente en términos políticos o bélicos, sino acompañándonos como seres humanos en la cotidianidad (HaroHonrubia, 2012).

El Poder ocupa un papel primordial dentro de las teorías del conflicto (Silva, 2008), y ha sido un objeto de estudio ampliamente desarrollado por la filosofía y la sociología clásica y moderna. En el marco de la sociología moderna, Michael Foucault ha sido uno de los teóricos más revolucionarios del siglo pasado al momento de desentrañar el poder. Para él, los análisis tradicionales de los aparatos del Estado o del Capital no son suficientes para conocer por completo el campo de su ejercicio, ni responden a las preguntas de quién, dónde, y hasta qué niveles se ejerce. Foucault afirma que el poder se ejercita a través de una organización reticular, donde cada lucha se desarrolla alrededor de un núcleo particular de poder (Foucault, 1979). Surge con esta idea la "microfísica del poder", donde el poder se entiende ya no desde las grandes instituciones sociales o clases dominantes, sino en las múltiples relaciones entre los individuos, y se ejercita dentro de una red compleja y multidimensional, en una u otra dirección dependiendo de quién ostente de los recursos necesarios para ejercerlo. Se establece una relación ambigua entre el poder y la vida cotidiana de los individuos, y para referirse a ella Foucault utiliza el término de Biopolítica, donde plantea que la ideología requiere del control del cuerpo del individuo. 
Comprenderemos el poder entonces no en un plano abstracto o simbólico, sino en el campo de la materialidad y la acción, como el control de un individuo o un grupo de individuos sobre las acciones de otro individuo o grupo distinto al primero. Tampoco entendemos el poder como una forma de represión, como ha sido utilizado históricamente, pues si el poder fuera únicamente represivo, es muy poco probable que sea obedecido. El poder debe comprenderse como una red productiva, que genera cosas, placer, saber y discursos (Foucault, 2001b).

Serrano-García y López-Sánchez (1991) ahondan en las relaciones de poder desde las Teorías del Conflicto. Para ellos, las relaciones de poder se ejercen cuando existe una base material asimétrica, es decir, cuando dos agentes están en conflicto por un recurso que uno controla, y al otro le interesa. Se entiende como recurso cualquier elemento - material o abstracto - disponible en una sociedad para la satisfacción de necesidades o aspiraciones humanas. El estado de asimetría de la relación no es un atributo fijo ni anterior al conflicto, sino que se desarrolla a través de un proceso psicológico en el que se va aumentando el nivel de conciencia sobre el estado del recurso en conflicto. Los autores definen cuatro niveles de conciencia, los cuales se resumen en el cuadro 1.

Cuadro 1. Niveles de conciencia de las asimetrías

\begin{tabular}{|c|c|c|}
\hline $\begin{array}{c}\text { NIVEL DE } \\
\text { CONCIENCIA }\end{array}$ & $\begin{array}{l}\text { ESTADO DEL } \\
\text { RECURSO }\end{array}$ & DESCRIPCIÓN \\
\hline Sumisa & Asimetría & $\begin{array}{c}\text { Realidad normalizada, natural, dada e } \\
\text { incambiable. }\end{array}$ \\
\hline Precrítica & Desigualdad & $\begin{array}{l}\text { Primeros atisbos de insatisfacción y } \\
\text { resentimiento confuso. Se comienza a buscar } \\
\text { explicaciones y causalidades. }\end{array}$ \\
\hline Crítica integradora & Injusticia & $\begin{array}{l}\text { Se comienza a analizar con precisión y } \\
\text { racionalidad la realidad social y comienzan } \\
\text { acciones de cambio. }\end{array}$ \\
\hline Crítica Liberadora & Opresión & $\begin{array}{l}\text { Comienza un proceso de transformación } \\
\text { social total. }\end{array}$ \\
\hline
\end{tabular}

Fuente: Elaboración propia a partir de Serrano-García y López-Sánchez (1991).

Va surgiendo así el interés por controlar el recurso que no se tiene, pues se desarrolla internamente una conciencia de que el recurso podría satisfacer necesidades y/o aspiraciones, y que a pesar de que otro agente lo controla actualmente, el recurso o su control sería transferible. Se trata de un conflicto interno, o como diría Festinger (1975) una disonancia cognoscitiva entre los ideales del docente y la realidad, que ahora se percibe incómoda, y que requiere ser cambiada. Este conflicto interno es el primer paso hacia la innovación educativa, y después de una reflexión crítica al respecto, se toma conciencia de que es necesario cambiar, pero que para ello primero se debe tener un mayor control sobre el recurso, que en este caso se configura como la acción pedagógica.

En este punto surge una transición de un proceso interno de toma de conciencia hacia un proceso externo donde el conflicto se materializa y se vuelve observable. El docente manifestará su deseo de cambiar su acción pedagógica y modificar los procesos de enseñanza-aprendizaje, y tendrá que enfrentarse a un sistema que a través de diversas herramientas lucha por mantener el control de esta acción. Mientras que el docente deberá ser consciente y hacer uso de sus propios recursos para ganar en esta lucha -como el conocimiento cercano de sus estudiantes, el saber técnico-pedagógico o el apoyo del colectivo docente-, los agentes externos pueden tener diversas tecnologías que les permitirán mantener o incluso aumentar el control sobre la acción pedagógica. A simple 
vista el control de los agentes externos resulta difuso, pues tal como señala Ball (2003) la combinación entre una instalada lógica de mercado, un marcado gerencialismo y rígidos mecanismos de performatividad sobre la que se basa la política educativa contemporánea conlleva a que los procesos educativos se manifiesten como procesos autónomos sin ningún tipo de regulación, pero que en realidad implica una red de sistemas de monitoreo y producción de información que logra cumplir un objetivo oculto de regulación y control de las escuelas. Como mencionamos anteriormente, son el currículum central y los procesos de accountability las tecnologías que se configuran como piezas claves en esta red de monitoreo que, ocultas bajo un discurso de autonomía, mantienen el control sobre la acción pedagógica.

Con todo esto, parece importante analizar la influencia ambos elementos como herramientas utilizadas para la resolución de un conflicto y el alcance de un consenso, pues tanto el conflicto como el consenso se definen como los subprocesos de un ajuste o cambio que a su vez permite al sistema su continuación en el tiempo (Alfaro y Cruz, 2010), y todo elemento implicado en el proceso de conflicto puede tener implicancias en la manera en que se llega a este consenso y, por lo tanto, la manera en que el sistema se ajusta.

\section{Metodología}

El objetivo de la presente investigación contiene dos condiciones que exigen entender tanto el conocimiento como la educación desde un enfoque crítico. Primero, porque se asume que los docentes son los principales - aunque no los únicos -agentes de cambio y que, por lo tanto, su propio empoderamiento y las relaciones de poder que se ejercen sobre ellos deberían tener efectos en los procesos de innovación (Escudero, 2014). Segundo, porque los paradigmas que le preceden entienden al investigador desde una posición asimétrica de experto que conoce e interpreta la realidad descrita por los sujetos investigados, ejerciendo un poder sobre los últimos al tomar sus voces subsumirlas a las propias tecnologías de representación (Balasch y Montenegro, 2003). Reproducir la tradicional posición asimétrica del investigador no sería coherente con un estudio que pretende, entre otras cosas, develar las relaciones de poder que se ejercen sobre los sujetos de estudio. El paradigma crítico y las metodologías que adopta intentan justamente reducir dicha asimetría de poder existente entre investigador y participante. Esto no significa, de todas maneras, que no asumamos claramente nuestro rol de investigadores como potenciales responsables de la generación del conocimiento en el contexto de este estudio. Por el contrario, se trata de asumir y transparentar dicho rol, revelando las ideas preconcebidas que motivan y orientan el estudio -ya descritas en el marco teórico-, pero al mismo tiempo haciéndonos cargo de las limitaciones de otros paradigmas, haciendo mucho más partícipes a los docentes en esta generación de conocimiento.

Con todo esto, se ha optado por realizar Producciones Narrativas, una técnica de investigación participativa derivada de la tradición antropológica y que ha tomado fuerza en la investigación psicosocial, por considerar fases de continuo diálogo entre investigadores e investigados, y donde los últimos tienen un protagonismo mucho mayor (Biglia y Bonet-Martí, 2009). En concreto, con esta técnica se construye un relato -con una temática propuesta y gatillada por el investigador- que se va revisando en constante diálogo entre investigador y participante, hasta llegar a un producto final que luego de su aceptación por ambas partes puede ser analizado bajo las técnicas tradicionales de investigación cualitativa (Balasch y Montenegro, 2003). Ésta técnica presenta dos 
ventajas coherentes con el enfoque adoptado. Por una parte, como técnica cualitativa permite recoger la voz de los docentes de forma profunda e inductiva, recogiendo categorías emergentes y reduciendo categorías preconcebidas por los investigadores. Por otra parte, como técnica participativa reduce la asimetría de poder entre investigador y participante, dándole mayor protagonismo a los últimos sobre sus propios discursos, favoreciendo el empoderamiento docente y dando consistencia al enfoque crítico en el que se enmarca el estudio.

Para esta investigación se ha contactado a un total de 9 profesores, todos de distintos establecimientos de la Región Metropolitana y la V Región de Valparaíso. La selección de los docentes responde a un muestreo intencionado de acuerdo a dos variables principales:

1. Dependencia Administrativa del establecimiento en el que trabajan: Pudiendo ser Municipal (Público), Particular Subvencionado (Privados que reciben subvención estatal directa) o Particular Pagado (Privados que no reciben subvención estatal directa).

2. Disciplina que imparten: Pudiendo ser Educación General Básica (que imparten varias disciplinas), Enseñanza Media de Asignatura (Lenguaje, Matemática, Ciencias Sociales o Ciencias Naturales) o Enseñanza Media de Especialidad (todas las otras disciplinas que no son evaluadas normalmente por SIMCE o PSU) ${ }^{1}$.

La elección de dichas categorías se basa en la presunción de que ambas pueden mostrar diferencias importantes en como los procesos de accountability inciden en la acción docente, pues de acuerdo al tipo de establecimiento responden a contextos y procesos burocráticos distintos, y de acuerdo a la asignatura son evaluados de diferentes maneras.

Con estos criterios, se ha diseñado una matriz donde se combinan tres docentes de cada tipo de establecimiento con tres docentes de cada grupo disciplinar. De esta manera, participaron 9 docentes que estaban en ejercicio en establecimientos chilenos durante las fechas en que construimos las narrativas, acomodándolos a los criterios establecidos. El cuadro 2 resume esta matriz.

Cuadro 2. Docentes participantes en Producciones Narrativas

\begin{tabular}{lccc}
\hline \multicolumn{1}{c}{ DEPENDENCIA } & \multicolumn{3}{c}{ DISCIPLINA } \\
\cline { 2 - 4 } ADMINISTRATIVA & Básica & Asignatura & Especialidad \\
\hline Municipal & Francisco & Manuel & Óscar \\
Subvencionado & Catalina & Josefina & Karina \\
Pagado & Rafael & Ignacio & Constanza \\
\hline
\end{tabular}

Fuente: Elaboración propia.

A los docentes se les invitó personalmente, sin intermediarios y sin la obligación de algún jefe o autoridad, y aclarando desde el inicio que la narrativa es totalmente confidencial, que será utilizada sólo en el contexto de este estudio y que sólo será utilizada la última versión aprobada por ambas partes. También se les dio la posibilidad de cambiar todos los nombres de pila y de instituciones dentro de la narrativa para asegurar su anonimato, opción que algunos profesores tomaron. Esto era muy importante para el estudio, pues los

\footnotetext{
1 Profesores de "Asignatura" o de "Especialidad" es una nomenclatura utilizada en la jerga de los docentes en Chile, y parece pertinente utilizarla considerando el enfoque participativo y crítico de este estudio.
} 
docentes debían hablar con total libertad y sin temor sobre distintos temas que incluían a sus jefes directos y el sistema educativo en su conjunto. Hemos explicado transparentemente el proceso al momento de contactarlos, aclarándoles que les significaría algo de tiempo. En algunos casos fue un factor que desmotivó la participación de docentes invitados, a pesar de que se les indicó que era una oportunidad para detenerse a revisar y reflexionar sobre sus propias prácticas profesionales y poder mejorarlas.

El proceso de construcción de las producciones narrativas se realizó durante el primer semestre de 2017. Para el primer encuentro con cada docente construimos una pauta de entrevista semiestructurada, la cual integraba diversas temáticas relacionadas con Innovación Educativa, Relaciones de Poder y Empoderamiento Docente. Esta entrevista dio paso a la escritura de un primer borrador con cada docente, el cual fue corregido y revisado varias veces en conjunto hasta llegar a un producto final. Cabe destacar que lo que se presentará aquí es parte de un estudio mayor, por lo que las narrativas consideraban muchos temas que no serán incluidos en este artículo.

Es importante recalcar, como se ha mencionado al inicio del apartado anterior, que el análisis de las producciones narrativas no se realiza "a la luz" del marco teórico, como lo hacen las técnicas tradicionales, sino en diálogo y articulación con el último. Este giro epistemológico no solo revaloriza al actor social, sino que además exige una segunda revisión del marco teórico, el cual debe complementarse con las nuevas miradas, haciendo un diálogo importante entre el saber experto y el experiencial para llegar a categorías de análisis adecuadas para la comprensión del fenómeno desde una perspectiva situada (Balasch y Montenegro, 2003). Por lo mismo, analizamos las narrativas a través de un proceso inductivo de codificación abierta y, una vez ordenadas las categorías, las complementamos con una nueva revisión de literatura coherente a los temas que vayan emergiendo. Se observará en la sección de resultados, por lo tanto, una amplia revisión de literatura que va a la par las categorías de análisis emergentes y que por los mismos motivos no ha sido expuesta en el apartado teórico.

\section{Resultados}

A continuación, revisamos los discursos de los docentes participantes en lo que respecta a su relación con el currículum central y los procesos nacionales de accountability, y cómo éstos afectan sus propios procesos de innovación educativa.

Lo primero que podemos observar en las narrativas es que, en muchos casos, los docentes se sienten atados a un sistema educativo que les impide tomar decisiones propias sobre cómo liderar las clases. La interacción del docente con el sistema está mediada casi siempre por el establecimiento educativo en el que trabajan, y está especialmente definida por el interés que tenga éste por cumplir con los indicadores impuestos y ceñirse a los estándares sugeridos por los programas del Ministerio de Educación.

Sobre el sistema curricular, en las narrativas encontramos discursos variados, con diversas problemáticas dignas de analizar. Por un lado, nos encontramos con docentes como Rafael, Francisco y Óscar, que afirman que el currículum los amarra con contenidos que no son los que ellos creen más pertinentes, pero que deben enseñar para no tener problemas a la hora de la fiscalización por parte de agentes internos o externos a su establecimiento. Esto es aún más fuerte cuando aparecen instituciones de asistencia técnica externa que instalan 
planes, programas y actividades absolutamente alineados con el currículum central, dejando poco espacio para hacer cosas distintas o innovadoras.

$Y$ a veces uno trata de salirse de esto, pero no es fácil, porque se deben pasar los contenidos y uno se mete en problemas si no los cumple. Una buena idea puede salir muy bien, pero si me quita tres o cuatro clases y no alcanzo a pasar los contenidos, ese costo lo pagaré tarde o temprano. (Rafael, Pagado, Básica)

En una línea que parece contraria, docentes como Francisco o Catalina mencionan que algunas instituciones de asistencia técnica externa instalan actividades que no son coherentes con el currículum. Éstas no llegan a transformar las aulas, sino que se añaden como una carga adicional a las actividades rutinarias, en lo que Astudillo e Imbarack (2013) llamarían un proceso de Fusión.

Ahora bien, ambos problemas mencionados son dos caras de una misma moneda. Ignacio y Josefina lo explican claramente: el problema no está en el currículum central, sino en la forma en que éste es interpretado por los establecimientos y los agentes fiscalizadores. En palabras de Josefina:

El currículum de lenguaje, sin ser todo lo coherente que a uno le gustaría, no está mal hecho. No está formulado en términos de contenido, como se tiende a pensar, sino de habilidades, $e$ intenta dar la libertad flexibilidad y libertad para orientar el programa. (...) El problema es cómo se lo toma la corporación y, principalmente, en cómo se evalúa su aplicación. Como tenemos que hacer pruebas cada mes, y dar indicadores duros, es imposible no hacer una evaluación parcelada, por lo que se reducen las habilidades lingüisticas «en contexto» a meros contenidos. Además, a veces me piden que evalúe indicadores que, si uno los lee bien, se da cuenta de que no están hechos para ser evaluados sumativamente, sino formativamente, en clases. $Y$ en algunos casos eso es muy problemático, porque hay muchos indicadores para los que tienes que inventar situaciones ficticias de evaluación en la que intentas hacer una clase normal, pero con nota, con una tabla de cotejo, y con los alumnos nerviosos porque están siendo evaluados mientras discuten, cuando la actividad está hecha para que discutan libremente. (Josefina, Subvencionado, Asignatura)

Algo similar menciona Ignacio sobre las bases curriculares de Historia, Geografía y Ciencias Sociales, pues normalmente superiores y supervisores revisan que se estén pasando los contenidos y realizando las actividades que se sugieren desde el Ministerio, mientras que las orientaciones oficiales dicen:

Se ha intentado aportar una batería significativa de recursos y actividades para que los docentes puedan seleccionar aquellos que mejor se adecúan a las necesidades y desafíos que enfrentan, considerando las diferencias individuales de sus estudiantes y la realidad de cada establecimiento. (...) en otras palabras, el docente debe seleccionar, modificar y adecuar las actividades de acuerdo a las necesidades que enfrenta. (MINEDUC 2012, p. 11)

Esta especificación, sin embargo, no se encuentra en otros documentos de otras disciplinas, por lo que es entendible que el currículum se tome al pie de la letra y de forma exhaustiva, como lo había sido tradicionalmente. Pero incluso muchos docentes de la asignatura mencionada tampoco saben de esta instrucción, lo que para Ignacio se explica en que está especificado en la introducción, y que los docentes comienzan a revisar las orientaciones a partir de los contenidos concretos, obviando esta parte que parece más protocolar.

Los profesores en general tienen dominio sobre su disciplina, pero no sobre el currículum. Porque un profesor empoderado que se leyó y apropió del currículum puede decirle a su jefe de UTP «Mira esta página, ¿la leíste? Dice clarito SELECCIONAR contenidos. (Ignacio, Pagado, Asignatura) 
Pero asociado al currículum existe una limitante aun mayor, que tiene que ver con los procesos de accountability del sistema chileno. En las narrativas se observan fuertes efectos de los procesos de accountability en diversos ámbitos del trabajo, tanto de las escuelas como de los docentes, aunque en el caso de los últimos, estos difieren de acuerdo con la situación del propio docente. Así, los profesores de enseñanza básica y media de asignatura sólo declaran sentirse presionados por la prueba SIMCE cuando les toca impartir clases en cursos que la rendirán ese mismo año, lo que llama profundamente la atención si se considera que la educación es un proceso holístico e integral. Pareciera que la prueba está generando más presión particular en docentes específicos que estrategias globales en los establecimientos educativos. Así mismo, los resultados PSU no son un indicador por el que los docentes de enseñanza básica se vean afectados, aunque tampoco por Manuel, profesor de Historia en enseñanza media, quién afirma que se ha traspasado la responsabilidad por los resultados PSU a los propios estudiantes y sus posibilidades de prepararla a través de preuniversitarios. Por el contrario, Ignacio dice no estar tan condicionado por SIMCE como lo está por la PSU, lo que sugiere que en los niveles socioeconómicos más acomodados hay mayor presión para la escuela sobre el éxito de los estudiantes en esta prueba.

Externamente, los profesores tenemos mucha presión por la prueba SIMCE -y no tanto por la PSU, que es un tema más privatizado y la gran mayoría los estudiantes están en preuniversitario-. A mi personalmente no me toca, porque no le hago clases a cursos que rindan SIMCE. (Manuel, Municipal, Asignatura)

Los efectos de estas pruebas percibidos por los docentes pueden ser englobados en un concepto clave: estrechamiento Curricular. El estrechamiento curricular -también llamado Reducción o Empobrecimiento Curricular- es un efecto asociado y al menos no explicitado como intencional de las pruebas estandarizadas de alto impacto, en el cual los establecimientos educativos- especialmente los más vulnerados- centran sus recursos materiales y temporales en los contenidos del currículum que serán evaluados, prestando poca o nula atención a otros contenidos y objetivos del currículum y perdiéndose la posibilidad de una formación más integral de los estudiantes (Berliner, 2011). En las narrativas observamos el estrechamiento curricular dentro de los establecimientos de dos maneras: intradisciplinario e interdisciplinario.

El estrechamiento curricular es intradisciplinario porque, dentro de las disciplinas que evalúan las pruebas estandarizadas -Lenguaje, Matemáticas, Ciencias Sociales y Ciencias Naturales- se presiona a los docentes por centrar la enseñanza en contenidos y habilidades SIMCE y PSU, no sólo enfocando los contenidos, sino destinando parte importante del tiempo, en especial durante el último mes antes de la rendición de la prueba, para actividades de adiestramiento, como pueden ser ensayos y rutinas SIMCE o PSU. Así, se va instalando dentro de las asignaturas un concepto de "Calidad" muy tecnocrático, que deja de lado la formación integral para centrarse en habilidades muy específicas que pueden medirse y representarse ordinalmente.

El profesor termina instrumentalizando al alumno para que rinda, porque es presionado por el colegio, por la corporación y por el Ministerio, que lo único que le importa es el puntaje que reciba $4^{\circ}$ básico. Este año me tocó ese curso, y ya los estamos machacando con ensayos, y la leyenda está puesta en la sala «Vamos que se puede: SIMCE 2017. (Francisco, Municipal, Básica)

Pero también es interdisciplinario, porque la presión de los centros por mejorar aprendizajes sólo está puesta hacia los docentes de las asignaturas evaluadas, en desmedro 
de las otras disciplinas. Los docentes de especialidad explican que a sus disciplinas no se las valora como a otras, y que están ahí como "accesorios", sólo porque el currículum nacional lo exige. Estos docentes tienen una libertad mucho mayor para tomar decisiones, pues tienen escasa supervisión y control, pero el costo de esta libertad es un total abandono que implica escasos lineamientos -más allá de los definidos por el currículum- y muy bajo apoyo por parte de los directivos y las autoridades. Quizás aquí es donde mejor se observa que las relaciones de poder también pueden ser necesarias y productivas (Foucault, 2001a), y que su ausencia también puede provocar situaciones desfavorables.

Como el colegio se mide por SIMCE o PSU, a nadie le interesa lo que hagamos. (Karina, Subvencionado, Especialidad)

Ahora bien, el accountability también tiene un efecto discursivo en los docentes, pues tal como afirma Au (2009), impacta en la propia identidad profesional de los profesores. Cuando hablamos de la identidad de los docentes, la entendemos relacionada a aquellas prácticas y procesos por los que los individuos hacen sentido y exhiben sus subjetividades en su espacio social (Rowsell y Abrams, 2011). Por lo tanto, entendemos la identidad no como no como una posesión del individuo, sino una puesta en acción a través de sus prácticas en sociedad y no como un atributo fijo de una persona, sino en tanto fenómeno flexible y relacional (De Tezanos, 2012).

Gran parte de la identidad docente en Chile se ve articulada por las categorías culturales del propio contexto escolar y las condiciones materiales de su trabajo, reflejadas tanto en los insumos de trabajo como en los sueldos percibidos (de Tezanos, 2012). Las identidades profesionales también se ven configuradas por la existencia de "otros", que permite la construcción del "sí mismo". Ávalos y Sotomayor (2012) plantean además que los docentes chilenos definen su identidad en referencia directa de sus alumnos, y que el reconocimiento de sus acciones depende en gran medida de la valoración que de ellos tengan los estudiantes, sus familias y, posiblemente, sus colegas.

Así, nos encontramos en las narrativas con docentes que han configurado su identidad a partir de todos estos elementos, desarrollando en su discurso docente "ideal", pero también un docente "real". En otras palabras, los docentes han configurado una idea de lo que debe ser el rol docente, pero también observan una realidad donde este rol se exige y se despliega de una forma diferente, lo que muchas veces entra en conflicto.

El docente ideal desempeña un rol de gestor y mediador de un conocimiento integral. Hacen mucho hincapié en que los docentes ya no pueden contentarse con transmitir el conocimiento, porque hoy este conocimiento se encuentra en todas partes, y que deben ser capaces de generar competencias en los estudiantes. También destacan que el docente debe formar en valores, enseñar a los estudiantes a ser "buenas personas" que sepan vivir y colaborar en comunidad, y que logren una combinación de pensamiento crítico y respeto que les permita desenvolverse y generar cambios en el futuro. Destacan que para lograr todo esto se debe tener muy presente el componente emocional, y motivar a los estudiantes parece ser uno de los objetivos principales. Por eso, se asocia mucho las innovaciones educativas a actividades que puedan motivar a los estudiantes.

En este sentido, la educación se debe basar en la motivación, pues es ésta la que gatilla procesos de pensamiento. Por muy racional que sea un contenido, si no se trabaja el componente emocional nunca se gatillará el aprendizaje. (Josefina, Subvencionado, Asignatura)

Sin embargo, también nos encontramos con un sentimiento de que las expectativas puestas sobre los docentes por parte del sistema y la sociedad no se condicen con este rol 
ideal. Según los docentes, el paradigma neoliberal imperante, especialmente reforzado por las evaluaciones de aprendizaje, ha tecnificado e instrumentalizado la educación, generando expectativas resultadistas y exitistas, sin interés por los procesos de enseñanza.

\begin{abstract}
Ahora al sistema le interesa sólo lo que se mide. Los valores están en el currículum, en los objetivos transversales, y uno trata de reforzarlo, pero eso te pasa la cuenta al final, porque tienes que rendir en lo otro, y si no eres un mal profesor, en una mala escuela. Los valores nadie los mide. (Francisco, Municipal, Básica)
\end{abstract}

Adicionalmente, el mismo currículum central deja de ser integral, por estar construido desde la división y atomización del trabajo, los contenidos y el pensamiento, todo en favor de la eficiencia. Y las asignaturas que más sufren con esto son las artísticas y humanistas, a las cuales no se les toma el peso por enfocarse en una formación de futuros trabajadores para el sistema y, por supuesto, en un buen rendimiento en las evaluaciones estandarizadas. Todo esto tiene un efecto directo en la identidad docente, pues comienzan a transformarse en técnicos de enseñanza para satisfacer las demandas del sistema, o cuestionan el verdadero sentido de su trabajo cuando éste no es tomado en cuenta, en el caso de los docentes de especialidad.

En contextos vulnerables (...) todo el mundo le da más importancia a los ramos que tienen pruebas estandarizadas. Es obvio, quieren rendir bien y que el colegio saque buen puntaje. Por eso sentía que Inglés era casi un accesorio, que sólo estaba porque se exigía en el currículum nacional. (Constanza, Pagado, Especialidad)

\title{
5. Discusión y Conclusiones
}

El sistema educativo chileno parece configurarse bajo un paradigma curricular aún muy tecnológico, es decir, bajo una lógica en que la enseñanza se comprende como un proceso único, controlable, fragmentable y lineal (Tejada, 2005). Tal y como ya advertía Au (2011) cuando nos hablaba de la aplicación del nuevo Taylorismo a la estructura, organización y currículum de los centros educativos, esto se traduce en que los docentes viven una experiencia de enseñanza artificialmente homogeneizada, difícil de cambiar o adaptar a los propios contextos locales, y regida bajo estándares parcelados, con poca interacción existente entre los contenidos, y aún menos entre contenidos de diferentes disciplinas. Todo esto se contradice bastante con las necesidades del siglo XXI, que requiere de individuos formados y con competencias para concebir globalmente las situaciones, y no sólo focalizadas en marcos específicos o fragmentados (Jonnaert, Barrette, Masciotra y Yaya, 2008).

Con todo esto, en esta era de estandarización del currículum (Gershon, 2017) los docentes se sienten atrapados bajo un sistema que les da poca libertad para explorar, equivocarse y aprender, y que poco propicia los espacios de reflexión crítica individual o colectiva sobre el quehacer pedagógico. El currículum central ya dice todo lo que debe saber y enseñar un docente, y los estándares establecidos indican el enfoque con que debe trabajarse. Las evaluaciones estandarizadas, en tanto, presionan a los docentes para que cumplan con este currículum, a través de consecuencias tanto directas como indirectas.

En este sentido, los docentes están en una desventaja importante al momento de realizar cambios e innovaciones, pues el sistema tiene herramientas poderosas para mantener el control de la enseñanza y reducir las posibilidades de un conflicto real que permita cambios profundos. Esto hace finalmente que las innovaciones que surgen sean de baja sostenibilidad e impacto, ya que se reducen a cambios superficiales en aspectos periféricos 
que no arriesguen los resultados de aprendizaje de los estudiantes que serán medidos en las evaluaciones estandarizadas.

La percepción del riesgo por parte de los docentes es un factor fundamental para comprender su implicación en procesos de innovación (Le Fevre, 2014). Pero no sólo es un tema de no tomar riesgos, sino que además el mismo sistema ha creado un contexto discursivo hipócrita, en el que los docentes se conciben como mediadores de un aprendizaje integral de los estudiantes y son invitados constantemente a innovar, mientras que el sistema burocrático respalda una visión del docente casi exclusiva como los principales responsables en la obtención de buenos resultados académicos, traducidos en puntajes SIMCE y PSU, visión que es adoptada por familias y sostenedores. Esta contradicción entre el rol percibido por los docentes y el concebido por el sistema educativo se condice con lo expresado por Fardella y Sisto (2015), quienes ven que las categorías discursivas prescritas por el sistema no se han introducido de manera natural ni han colonizado la experiencia docente, lo que se visualiza en el malestar y el cuestionamiento constante de dichas categorías. Con todo esto, las innovaciones profundas no sólo requieren de un esfuerzo técnico, sino sobre todo de un esfuerzo mayor en justificarlas y convencer a superiores y familias de que éstos serán beneficiosos en el largo plazo, esfuerzo que no trae beneficios mayores a los mismos docentes, por lo que finalmente decidirían no realizarlos.

Ahora bien, es necesario destacar que una de las principales limitaciones para innovar percibidas por algunos de los docentes no está en las mismas evaluaciones, sino en cómo son utilizadas por los fiscalizadores, y cómo han afectado la interpretación de los procesos educativos. En el fondo, el problema no está en lo que se mide, sino en cómo todo el proceso de accountability ha influido en la comprensión del currículum. Así, de acuerdo con los docentes, los fiscalizadores - superiores directos o supervisores de instituciones locales - confunden ciertas actividades evaluativas que tienen un carácter formativo y exigen realizarlas de forma sumativa, lo que afecta la calidad de los procesos de enseñanzaaprendizaje; o revisan que los contenidos del currículum central se estén dictando en las clases, a pesar de que el mismo currículum sugiera que no es necesario utilizar todos los contenidos, sino que debiesen ser seleccionados aquellos pertinentes para formar las competencias y habilidades planteadas como objetivos del establecimiento. Concordamos, por lo tanto, con Pritchett (2015) cuando afirma que un modelo de accountability exitoso requiere de una coherencia entre lo que se exige, lo que se observa, lo que se informa y los incentivos. Los supuestos controles de calidad establecidos de la manera en que se hacen terminan por impedir la mejora efectiva del sistema educativo y, por tanto, a la larga provocan un deterioro de esa calidad a la que pretenden servir.

En la otra cara de la misma moneda, el mismo sistema también afecta de forma negativa a los docentes de especialidad, es decir, aquellos que no son evaluados directamente por las evaluaciones estandarizadas. Éstos docentes ven un proceso de instrumentalización de las escuelas y de los estudiantes, y de focalización de los esfuerzos en las áreas evaluadas. Lo que no se mide, no vale. Los docentes de especialidad sienten un real abandono por parte de los líderes de su institución, lo que por un lado les da completa libertad para innovar, dentro de un contex to poco propicio para que dichas innovaciones sean reconocidas o se vuelvan sostenibles. Esto es realmente problemático cuando se considera que el objetivo principal del liderazgo pedagógico es mejorar la práctica docente y crear un entorno para el aprendizaje focalizado en expectativas claras para todos los profesores (Elmore, 2010). En ese sentido, se vuelve primordial entender que el poder no sólo es represivo, sino que también puede ser productivo en tanto las relaciones se desarrollan en equilibrio y 
consenso entre las partes, y que donde el conflicto es el motor del cambio, la ausencia de relaciones de poder reduce las posibilidades de innovación educativa.

Los resultados de este estudio parecen coherentes con estudios previos en los que se destaca la importancia de promover la agencia del profesorado para promover la innovación educativa (Astudillo e Imbarack, 2013; Farris-Berg, 2014; Vähäsantanen, 2015). Con todo, parece ser necesario que los establecimientos hagan más partícipes a todos sus docentes en las tomas de decisiones, y especialmente en el planteamiento de los objetivos, para que éstos se acerquen a los ideales de los docentes. Adicionalmente, es necesario que los docentes se empoderen, es decir, tomen el control sobre sus propias acciones (Montero, 2010), y sobre todo que de apoderen del currículum, de manera de poder utilizarlo a su favor como herramienta de cambio, y no como herramienta de opresión, como es percibida hoy en día.

Cabe destacar que este estudio tiene las limitaciones propias de cualquier estudio de carácter cualitativo, donde recogemos y analizamos con mucha profundidad las experiencias de los docentes, pero no son resultados generalizables y son difícilmente replicables, sobre todo considerando que sólo participaron docentes de la zona central de Chile. De todas maneras, la literatura sugiere que ésta no es una situación exclusiva del contex to chileno, y que en la política educativa internacional existe una tensión constante entre países que aumentar el control sobre el trabajo del profesorado, y aquellos que quieren promover su autonomía (Biesta, Priestley y Robinson, 2015). Con todo, parece importante seguir realizando investigación bajo diversas perspectivas metodológicas que aborden la innovación educativa desde las lógicas del conflicto y poder, entendiendo que el trabajo docente no es impermeable a los mandatos y expectativas burocráticas y sociales, y que todo intento de mejora se verá afectado por las relaciones de poder de las cuales son parte.

\section{Agradecimientos}

Este trabajo ha sido financiado por CONICYT PFCHA/DOCTORADO BECAS CHILE/2015 - Folio 72160138.

\section{Referencias Bibliográficas}

Alfaro, R. y Cruz, O. (2010). Teoria del cambio social y posmodernidad. Revista de Ciencias Sociales, 128-129, 63-70.

Assaél, J., Albornoz, N. y Caro, M. (2018). Estandarización educativa en Chile: tensiones y consecuencias para el trabajo docente. Educação Unisinos, 22(1), 83-90.

Astudillo, G. y Imbarack, P. (2013). El sentido del mejoramiento escolar de los docentes en el contexto de políticas de apoyo externo. Pensamiento Educativo. Revista de Investigación Educacional Latinoamericana, 50(2), 132-146.

$\mathrm{Au}, \mathrm{W}$. (2009). Unequal by design. High-stakes testing and the standardization of inequality. Nueva York, NY: Routledge.

Au, W. (2011). Teaching under the new taylorism: High-stakes testing and the standardization of the 21stcentury curriculum. Journal of Curriculum Studies, 43(1), 25-45. https://doi.org/10.1080/00220272.2010.521261 
Avalos, B. y Sotomayor, C. (2012). Cómo ven su identidad los docentes chilenos. Perspectiva Educacional, 51(1), 77-95. https://doi.org/10.4151/07189729-Vol.51-Iss.1-Art.74

Balasch, M. y Montenegro, M. (2003). Una propuesta metodológica desde la epistemología de los conocimientos situados: Las producciones narrativas. Encuentros en Psicología Social, 1(3), $44-48$.

Ball, S. J. (2003). The teacher's soul and the terrors of performativity. Journal of Education Policy, 18(2), 215-228. https://doi.org/10.1080/0268093022000043065

Berliner, D. (2011). Rational responses to high stakes testing: The case of curriculum narrowing and the harm that follows. Cambridge Journal of Education, 411(3), 287-302.

Biesta, G., Priestley, M. y Robinson, S. (2015). The role of beliefs in teacher agency. Teachers and Teaching: Theory and Practice, 21(6), 624-640. https://doi.org/10.1080/13540602.2015.1044325

Biglia, B. y Bonet-Martí, J. (2009). La construcción de narrativas como método de investigación psico-social. Prácticas de escritura compartida. Forum: Qualitative Social Research, 1O(1), art 8.

Burns, T. y Köster, F. (2016). Governing education in a complex world. Paris: OECD Publishing.

Casassus, J. (2010). Las reformas basadas en estándares: Un camino equivocado. En C. Bellei, D. Contreras y J. P. Valenzuela (Eds.), Ecos de la Revolución Pingüina (pp. 85-109). Santiago de Chile: Pehuén.

Cruz, R. y Croda, G. (2017). Concepciones sobre innovación educativa: Elementos para su teorización. Actas del Congreso Nacional de Investigación Educativa - COMIE (pp. 1-10). San Luis Potosí: COMIE.

De Tezanos, A. (2012). ¿Identidad y/o tradición docente? apuntes para una discusión. Perspectiva Educacional, 51(1), 1-28.

Durkheim, E. (1987). La división del trabajo social. Madrid: Akal.

Elmore, R. (2010). Mejorando la escuela desde la sala de clases. Santiago de Chile: Fundación Chile.

Escudero, J. M. (2014). Avances y retos en la promoción de la innovación en los centros educativos. Educar, 35, 101-138.

Espinola, V. y Claro, J. P. (2010). El sistema nacional de aseguramiento de la calidad: Una reforma basada en estándares. En C. Bellei, D. Contreras, y J. P. Valenzuela (Eds.), Ecos de la Revolución Pingüina (pp. 51-83). Santiago de Chile: Pehuén.

Falabella, A. y De La Vega, L. (2016). Políticas de responsabilización por desempeño escolar: Un debate a partir de la literatura internacional y el caso chileno. Estudios Pedagógicos, 42(2), 395-413.

Fardella, C. y Sisto, V. (2015). Nuevas regulaciones del trabajo docente en Chile. Discurso, subjetividad y resistencia. Psicologia y Sociedade, 27(1), 68-79.

Farris-Berg, K. (2014). A different model for school success: Empower teachers. Phi Delta Kappan, 95(7), 31-36. https://doi.org/10.1177/003172171409500707

Festinger, L. (1975). Teoría de la Disonancia Cognoscitiva. Madrid: Instituto de Estudios Políticos.

Foucault, M. (1979). Microfísica del Poder. Madrid: Las Ediciones de La Piqueta.

Foucault, M. (2001a). Poderes y estrategias. En M. Foucault (Ed.), Un diálogo sobre el poder y otras conversaciones (pp. 88-101). Madrid: Alianza. 
Foucault, M. (2001b). Verdad y poder. Diálogo con M. Fontana. En M. Foucault (Ed.), Un diálogo sobre el poder y otras conversaciones (pp. 139-156). Madrid: Alianza.

Gershon, W. (2017). Curriculum and students in classrooms: Everyday urban education in an era of standardization. Nueva York, NY: Lexinton Books.

Giles, C. y Hargreaves, A. (2006). The sustainability of innovative schools as learning organizations and professional learning communities during standardized reform. Educational Administration Quarterly, 42(1), 124-156. https://doi.org/10.1177/0013161X05278189

Haro-Honrubia, A. (2012). Antropología del conflicto. Reflexiones sobre el nuevo orden global. Convergencia. Revista de Ciencias Sociales, 19(60), 177-204.

Jonnaert, P., Barrette, J., Masciotra, D. y Yaya, M. (2008). La competencia como organizadora de los programas de formación: hacia un desempeño competente. Profesorado. Revista de Currículum y Formación del Profesorado, $12(3), 1-32$.

Kezar, A. J. (2001). Understanding and facilitating organizational change in the 21st century. San Francisco, CA: Jossey-Bass.

Le Fevre, D. M. (2014). Barriers to implementing pedagogical change: The role of teachers' perceptions of risk. Teaching and Teacher Education, 38, 56-64. https://doi.org/10.1016/j.tate.2013.11.007

Margalef, L. y Arenas, A. (2006). ¿Qué entendemos por innovación educativa? A propósito del desarrollo curricular. Perspectiva Educacional, 47, 13-31.

McMeekin, R. W. (2006). Hacia una comprensión de la accountability educativa y cómo puede aplicarse en los países de América Latina. En J. Corvalán y R. W. McMeekin (Eds.), Accountability educacional: Posibilidades y desafíos para América Latina a partir de la experiencia internacional (pp. 19-50). Santiago de Chile: PREAL-CIDE.

MINEDUC. (2017). Sistema Curricular. Recuperado de http://www.curriculumnacional.cl/sistema-curricular/

MINEDUC. (2012). Orientaciones Generales para Historia, Geografía y Ciencias Sociales de 1 o a 60 Básico. Recuperado de hhttp://www.curriculumnacional.cl/inicio/1b-6b/sextobasico/historia-geografia-y-ciencias-sociales/

Montero, M. (2010). Fortalecimiento de la ciudadanía y transformación social: Área de encuentro entre la psicología política y la psicología comunitaria. Psykhe, 19(2), 51-63. https://doi.org/10.4067/So7 18-2228201000020000198

Murillo, F. J. (2002). La «mejora de la escuela»: Concepto y caracterización. En F. J. Murillo y M. Muñoz-Repiso (Eds.), La mejora de la escuela (pp. 15-51). Barcelona: Octaedro.

OCDE. (2013). Learning standards, teaching standards and standards for school principals. París: OCDE.

Parsons, T. (1999). El sistema social. Madrid: Alianza.

Pritchett, L. (2015). Creating education systems coherent for learning outcomes: Making the transition from schooling to learning. Oxford: RISE.

Puryear, J. (2006). La accountability en la educación: ¿Qué hemos aprendido? En J. Corvalán y R. McMeekin (Eds.), Accountability educacional: Posibilidades y desafíos para América Latina a partir de la experiencia internacional (pp. 125-134). Santiago de Chile: PREAL-CIDE.

Rivas, M. (2000). Innovación educativa. Teoría, procesos y estrategias. Madrid: Síntesis. 
Rowsell, J. y Abrams, S. (2011). (Re)conceptualizing I/identity: An introduction. Teachers College Record, 113(13), 1-16.

Sánchez Chacón, G. (2016). Percepción sistémica de la innovación educativa : Reflexiones desde el nuevo paradigma científico. Revista Ensayos Pedagógigos, 11(1), 17-39.

Serrano-García, I. y López-Sánchez, G. (1991). Una perspectiva diferente del poder y el cambio social para la psicología social-comunitaria. Revista de Ciencias Sociales, 29(3-4), 349-381.

Silva, G. (2008). La teoría del conflicto. Un marco teórico necesario. Prolegómenos. Derechos y Valores, $11(22)$, 29-43.

Tejada, J. (2005). Didáctica-Currículum: diseño, derarrollo y evaluación curricular. Barcelona: Davinci.

Vähäsantanen, K. (2015). Professional agency in the stream of change: Understanding educational change and teachers' professional identities. Teaching and Teacher Education, 47(Abril), 1https://doi.org/12.10.1016/j.tate.2014.11.006

\section{Breve CV de los autores}

\section{Javier Pascual Medina}

Sociólogo de la P. Universidad Católica de Chile, Máster en Investigación en Educación con mención en Currículum y Procesos de Innovación de la Universidad Autónoma de Barcelona e Doctorando en Educación por el Departamento de Pedagogía Aplicada de la Universidad Autónoma de Barcelona. Ha trabajado como investigador y coordinador de proyectos de investigación en el Área de Estudios del Centro de Innovación en Educación de Fundación Chile, y como colaborador en el Equipo de Investigación en Diversidad e Inclusión en Sociedades Complejas (ERDISC). Sus investigaciones previas se han enfocado en temas como liderazgo educativo, innovación educativa, política educativa, docencia y evaluación y medición de aprendizajes. ORCID ID: 0000-0001-6850-7391. Email: jpascualmedina@gmail.com

\section{David Rodríguez Gómez}

Profesor del Departamento de Pedagogía Aplicada de la Universitat Autònoma de Barcelona (UAB) e investigador del Equipo de Desarrollo Organizativo (http://edo.uab.cat). Desarrolla su actividad docente principal, de grado y posgrado, como profesor de la Facultad de Ciencias de la Educación de la UAB, además de ejercer como profesor colaborador de la Universitat Oberta de Catalunya. La investigación del profesor Rodríguez-Gómez se centra, entre otros aspectos, en el estudio de los procesos de gestión del conocimiento en las organizaciones, vinculándolo a procesos de aprendizaje informal y social, así como al desarrollo y mejora de las organizaciones; en el estudio del abandono y retención de los estudiantes universitarios; y en el uso de las TIC a los centros educativos. ORCID ID: 0000-0001-9845-0744. Email: david.rodriguez.gomez@uab.cat 